\title{
Alternative Werkstoffe für Tragmittel in der Förder- und Materialflusstechnik
}

\author{
DR.-ING. WOLFRAM VOGEL, \\ DR.-ING. SILKE SCHÖNHERR \\ INSTITUT FÜR FÖRDERTECHNIK UND LOGISTIK, UNIVERSITÄT STUTTGART
}

Zusammenfassung

Die Anforderungen an laufende Tragmittel in Seiltrieben in der Förder- und Materialflusstechnik sind vielfältig, wie z.B. beim Einsatz in aggressiven und korrosiven Umgebungen. Der Einsatz von nicht rostenden laufenden Edelstahlseilen drängt sich für solche Anwendungen förmlich auf. Allerdings können die bekannten Dimensionierungsregeln von über Scheiben laufenden Tragmitteln aus Drähten aus nicht legierten Kohlenstoffstählen nicht auf Edelstahlseile übertragen werden. An Beispielen aus der industriellen Anwendung in Lackier- und Entfettungsanlagen und bei Schleusentoren in der Binnenschifffahrt konnte aufgezeigt werden, dass sich die laufenden Edelstahlseile qualitativ hinsichtlich der Lebensdauer ähnlich verhalten wie die bereits angesprochenen herkömmlichen Tragmittel. Allerdings ist die Lebensdauer bei laufenden Edelstahlseilen um den Faktor 3 - 8 kleiner als bei Tragmitteln aus Drähten aus unlegierten Kohlenstoffstählen. Diese Erkenntnisse und Erfahrungen sind genutzt worden, um eine grundlegende vorwettbewerbliche Forschung mit dem Titel „Laufende Edelstahlseile“ anzustoßen, die durch die Industrie und aus Haushaltsmitteln des Bundesministerium für Wirtschaft und Arbeit BMWA über die Arbeitsgemeinschaft industrieller Forschungsvereinigungen „Otto von Gericke“ e.V. (AiF) gefördert wird. Über erste Ergebnisse zu Lebensdauer und Ablegereife von laufenden Edelstahlseilen, ermittelt in systematischen Dauerbiegeversuchen, bei denen die maßgebenden Parameter (Seilzugkraft, Durchmesserverhältnis, Konstruktion, Schmierung etc.) in weiten Grenzen variiert werden, wird hier berichtet.

\begin{abstract}
The demands on running suspension means in rope drives within material handling and materials flow technology are manifold, as, e.g., when used in aggressive environmental and corrosive surroundings. Thus, the usage of non-corrosive running stainless steel ropes suggests itself for corresponding applications. It is, however, the case that established dimensioning rules for load bearing wire elements running over sheaves and constructed of unalloyed carbon steels cannot be applied to stainless steel ropes. Examples from industrial applications in varnishing and degreasing installations and from sluice gates in inland navigation were suited to demonstrate that running stainless steel ropes exhibit similar qualitative properties with a view to their lifetime as aforementioned traditional load bearing elements. However, lifetime in running stainless steel ropes is reduced by a factor of 3 to 8 in comparison with load bearing wire elements of unalloyed carbon steels. The results and experience thus obtained were applied to initiate a precompetitive foundational research project entitled "Running Stainless Steel Ropes" funded by industry and through budgetary funds from the 'Bundesministerium für Wirtschaft und Technologie BMWI (Federal Ministry of Economics and Technology) through the 'Arbeitsgemeinschaft industrieller Forschungsvereinigungen “Otto von Guericke e.V.” (German Federation for Industrial Research Associations “Otto von Guericke e.V.”) and by funds from the German Drahtseil-Vereinigung e.V. The article here presented sets out to explain initial results regarding lifetime and discard of running stainless steel ropes as investigated in systematic fatigue bending tests during which relevant parameters (tensile force, diameter ratio, construction, lubrication etc.) were varied within a wide range.
\end{abstract}

\section{Problemstellung}

So vielfältig wie die Ausprägung von fördertechnischen Systemen und Materialflusssystemen ist, so vielfältig sind auch die Anforderungen, die an sie gestellt werden hinsichtlich Arbeitsweise, Steuerung, Dimensionierung und vor allem Konstruktion der einzelnen Bauelemente und Baugruppen. Fördertechnische Systeme in Umgebungen wie z.B. der Lebensmittelindustrie, der Automobillackierung mit besonderen Anforderungen an die Reinheit, aber auch in Anwendungen, wo mit dem Einsatz von aggressiven Medien gerechnet werden muss, zeigen, dass Tragmittel aus herkömmlichen, unlegierten Stahlwerkstoffen nur sehr bedingt geeignet sind. Tragmittel aus hochfesten Kunstfasern, Riemen mit Einlagecords aus Stahl und/oder Fasern, aber auch Tragmittel aus Drähten aus Edelstählen finden hier ihre besondere Anwendung. Edelstahlseile mit Drähten, meist aus den 
Werkstoffen 1.4401, in einigen Fällen auch 1.4436, kommen dann in Seiltrieben der fördertechnischen Systeme „über Scheiben laufend“ zum Einsatz, wenn erhöhter Seilverschleiß durch Korrosion aufgrund von aggressiven Umgebungsbedingungen vorliegt oder zu erwarten ist. An diese laufenden Tragmittel werden aber unabhängig vom Drahtwerkstoff sicherheitsrelevante Anforderungen nach ausreichender Lebensdauer und rechtzeitiger Erkennung der Ablegereife gestellt, bevor ein gefährlicher Zustand für Mensch und Sachwerte entsteht.

Bei laufenden Seilen ist die Prognose der Seillebensdauer in den Seiltrieben von sicherheitsrelevanten Anwendungen von entscheidender Bedeutung. Die Seillebensdauer von Seilen mit Drähten aus unlegiertem Kohlenstoffstahl ist gut und punktuell bei hochfesten Faserseilen und Riemen mit Stahlcordeinlage zum Teil schon untersucht. Für Seile aus Edelstahldrähten trifft dies aber in keiner Weise zu. In jüngerer Vergangenheit sind an das Institut für Fördertechnik und Logistik vermehrt Anfragen zu den Lebensdauern von über Scheiben laufenden Edelstahlseilen in zum Teil sicherheitsrelevanten, fördertechnischen Anwendungen und auch in sensiblen Materialflusssystemen gestellt worden. Hierzu zählen die Schleusenantriebe des Main-Donau-Kanals, Seiltriebe in Kraftwerken, Türantriebe in Personenkraftwagen und Seiltriebe in Entfettungsanlagen und Lackierstraßen bei der Automobilherstellung etc. Es musste leider festgestellt werden, dass Edelstahltragmittel in gleicher Weise betrachtet und dimensioniert wurden wie herkömmliche Tagmittel aus unlegierten Kohlenstoffstahldrähten. Es blieb unberücksichtigt, dass die Dauerfestigkeiten sowohl bei Zugschwellbeanspruchung als auch der Dauerbiegewechselbeanspruchung hinter den herkömmlichen Tragmitteln zurückbleiben. Nur durch massiven versuchstechnischen Aufwand und aufwendige Umkonstruktionen konnten in den genannten Fällen gefährliche Situationen im Vorfeld der Inbetriebnahme abgewendet und/oder wirtschaftlicher Schaden minimiert werden. Somit entsteht an dieser Stelle eine sicherheitsrelevante und wirtschaftliche Lücke, die durch ein Forschungsvorhaben geschlossen werden soll. Im folgenden Papier wird über die ersten Ergebnisse von Dauerbiegeversuchen an ausgewählten Edelstahlseilen, die in fördertechnischen Anlagen und Materialflusssystemen zum Einsatz kommen, berichtet. Die statistische Auswertung der Biegewechselzahl von Edelstahlseilen wird stets verglichen mit den Ergebnissen, die mit herkömmlichen Tragmitteln ermittelt werden können. Ziel des Vorhabens ist die Bereitstellung von Berechnungsgrundlagen für laufende Edelstahlseile in beliebigen Fördertechnik- und Materialflusssystemen.

\section{Beispiel 1 - Lebensdauerprognose von Edelstahlseilen in den Seiltrieben einer Nassanlage zur Karosserievorberei- tung/-lackierung}

In der Automobilindustrie ist ein wesentlicher Prozessschritt in der Nassanlagen-Karosserielackierung und vorbereitung zu sehen. Diese Nassanlagen sind regelmäßig in Abschnitte unterteilt, die durch geteilte Schiebetore, z.B. Teleskoptüren mit 2 Türblättern, voneinander getrennt sind. Die beiden Türblätter sind praktisch immer durch einen Seiltrieb miteinander verbunden.

Wegen der chemisch aggressiven Umgebung sind die Abschnitte der Nassanlagen und auch die Türblätter der Teleskoptür, der Seiltrieb und die Seile aus nicht-rostendem Material bzw. nichtrostenden Drähten hergestellt. Bei verschiedenen Teleskoptüren ist es zu einem unerwartet frühen Seilausfall und damit Seilwechsel mit Stillstandszeiten gekommen. Damit hat ein nicht beachtetes „Seilchen“ die gesamte Produktion ins Stocken gebracht. Somit besteht dringender Handlungs- und Forschungsbedarf.

\subsection{Seile}

In den Teleskoptüren waren Seile der Konstruktion 6x19 Standard mit Stahleinlage und einem Nenndurchmesser von d = 3 mm eingesetzt. Die technischen Daten des Seils sind in Tabelle 1 zusammengefasst. Bei der Standardverseilung sind die Schlagwinkel der Drahtlagen gleich und die Schlaglängen unterschiedlich, mit der Folge von punktförmigen Drahtkontakten. Günstiger für laufende Seile sind Konstruktionen mit Litzen in Parallelschlag, d. h. die Drähte haben gleiche Schlaglänge und berühren sich linienförmig. Für die Lebensdaueroptimierung ist ein Seil Warrington 6x19 mit Stahleinlage gewählt worden, das in Edelstahl mit dem kleinen Durchmesser auch real verfügbar ist. Die technischen Daten dieses Seils sind ebenfalls in Tabelle 1 zusammengefasst. Wegen der aggressiven Umgebung sind die Seile aus Edelstahldrähten hergestellt. 
Tabelle 1: Technische Daten der beiden Edelstahlseile

\begin{tabular}{|l|c|c|}
\hline & $\begin{array}{c}\text { 6x19 Standard } \\
\text { und Stahleinlage }\end{array}$ & $\begin{array}{c}\text { Warrington 6x19 } \\
\text { und Stahleinlage }\end{array}$ \\
\hline Seilnenndurchmesser d $[\mathrm{mm}]$ & 3 & 3 \\
\hline Seil-Istdurchmesser d $[\mathrm{mm}]$ & 3,0 & 3,2 \\
\hline Nennfestigkeit $\mathrm{R}_{0}\left[\mathrm{~N} / \mathrm{mm}^{2}\right]$ & 1570 & 1570 \\
\hline Drahtwerkstoff & 1.4401 & 1.4401 \\
\hline Rech. Bruchkraft $\mathrm{F}_{\mathrm{r}}[\mathrm{KN}]$ & 5,84 & -- \\
\hline Mindestbruchkraft $\mathrm{F}_{\min }[\mathrm{KN}]$ & 4,66 & -- \\
\hline Schlagrichtung & rechtsgängig & rechtsgängig \\
\hline Schmierung & trocken & trocken \\
\hline
\end{tabular}

\subsection{Seiltrieb}

Der Seiltrieb besteht aus einer einseitig öffnenden Teleskoptür mit zwei Türblättern. Maßgebend ist dabei das Seil, das bei einem Spiel von der Trommel abläuft, dann gegensinnig gebogen über die Umlenkscheibe 1 läuft und nochmals gleichsinnig um Rolle 2 gebogen wird. Der zweite Seilabschnitt läuft auf die andere Seite der Trommel auf und entgeht somit der Gegenbiegung zwischen Trommel und Rolle 1. Die technischen Daten der Trommel und der Scheibe sind in Tabelle 2 zusammengefasst.

Tabelle 2: Daten des Seiltriebs

\begin{tabular}{|l|c|}
\hline Trommeldurchmesser $\mathrm{D}_{\mathrm{r}}$ (Seilmitte-Seilmitte) $[\mathrm{mm}]$ & 60 \\
\hline Rollendurchmesser $\mathrm{D}_{\mathrm{r}}($ Seilmitte - Seilmitte) $[\mathrm{mm}]$ & 60 \\
\hline Rillenradius Trommel $\mathrm{r}_{\mathrm{r}}[\mathrm{mm}]$ & 1,75 \\
\hline Rillenradius Scheibe $\mathrm{r}_{\mathrm{s}}[\mathrm{mm}]$ & $1,6^{*}$ \\
\hline Max. Schrägzug $\left.\varphi{ }^{\circ}{ }^{*}\right]$ & 3 \\
\hline
\end{tabular}

Das Motormoment ist in der Beharrung mit $\mathrm{M}=205 \mathrm{Ncm}$ und beim Anhalten mit M=1080 Ncm angegeben. Mit dem Trommelradius aus Tabelle 1 ist die Seilkraft S=70 N bzw. S=350 N. Um auf die sichere Seite zu kommen und wegen fehlender, genauer Angaben, wird hier mit der größeren Kraft und zusätzlich mit einer Seilzugkraft von $S=150 \mathrm{~N}$ gerechnet. Die Torgeschwindigkeit ist mit $\mathrm{v} \approx 0,4 \mathrm{~m} / \mathrm{s}$ klein. Änderungen der Seilzugkraft aus der Beschleunigung werden nicht gesondert berücksichtigt.

\subsection{Biegefolge beim bestehenden Seiltrieb}

Bei einem Hubspiel läuft das höchst beanspruchte Seilstück von der Trommel gegensinnig über Rolle 1 und gleichsinnig über Rolle 2 ab und entsprechend zurück. Die höchst beanspruchte Seilzone erfährt pro Hubspiel 2 Gegenbiegungen und 3 gleichsinnige Biegungen.

\subsection{Biegewechselzahlen}

Für die beiden in Tabelle 1 aufgelisteten Seile werden die Spielzahlen $\mathrm{Z}_{10}$ (Bruchspielwechselzahl, die mit einer Sicherheit von $95 \%$ von höchstens $10 \%$ der Seile nicht erreicht wird) und $\mathrm{Z}_{\mathrm{A} 10}$ (Ablegespielzahl, bei der mit Sicherheit von 95 \% höchstens 10 \% der Seile ablegereif sind) gesucht. Hier sei angemerkt, dass für die Nachrechnung $Z_{10}$ und für eine zukünftige Auslegung $Z_{\mathrm{A} 10}$ maßgebend ist. Die Spielzahlen sind in Tabelle 3 aufgelistet. Die Berechnungen werden mit folgenden Voraussetzungen durchgeführt: ungeschmierte Seile, Schrägzugwinkel von $3^{\circ}$, Lebensdauerabminderung Edelstahl Faktor $=3$ (geschätzt), durchgeführt. Auf eine Lebensdauerberechnung des Seils 6x19 Standard mit Stahleinlage wird explizit verzichtet. Die Lebensdauer ist aber mindestens um den Faktor 3 schlechter als beim Parallelschlagseil.

Tabelle 3: Berechnete Spielzahlen für das Seil Warrington 6x19 + Stahleinlage Schrägzug $3^{\circ}$, trocken, Edelstahl, $\mathrm{D}=60 \mathrm{~mm}$

\begin{tabular}{|l|c|c|c|c|}
\hline & \multicolumn{2}{|c|}{ Bruchspielzahl $\mathrm{Z}_{10}$} & \multicolumn{2}{c|}{ Ablegespielzahl $\mathrm{Z}_{\mathrm{A} 10}$} \\
\hline Seilzugkraft N & 150 & 350 & 150 & 350 \\
\hline Spiele & 2300 & 800 & 1200 & 400 \\
\hline
\end{tabular}

\footnotetext{
* für das Seil W 6x19 + Stahleinlage mit Seildurchmesser $\mathrm{d} \approx 3,2 \mathrm{~mm}$ sind Zwängungen nicht auszuschließen.
} 
Bei beiden Belastungen muss bei bestehendem Seiltrieb mit einem frühen Erreichen der Ablegereife gerechnet werden.

\subsection{Maßnahmen zur Steigerung der Seillebensdauer im bestehenden Seiltrieb}

Unter Beibehaltung des Seiltriebes kann die Lebensdauer der Seile gesteigert werden durch

- $\quad$ eine Schmierung, ggf. auch im Betrieb, wobei auf mit der Fertigung verträgliche Schmierstoffe zurückgegriffen werden sollte,

- $\quad$ Reduzierung des Schrägzuges zwischen Trommel und Rolle 1, z. B. durch Vergrößerung des Abstandes zwischen Trommel und Rolle 1,

- Vermeidung von scharfen Kanten, die z. B. bei der Herstellung der Trommel entstehen können,

- $\quad$ Vergrößerung der Trommel- und Scheibendurchmesser $\mathrm{D}_{\mathrm{T}}$ und $\mathrm{D}_{\mathrm{S}}$,

- $\quad$ Abstimmung der Rillenradien $\mathrm{r}_{\mathrm{T}}$ und $\mathrm{r}_{\mathrm{S}}$ auf den Ist-Durchmesser der Seile, mit dem Ziel, $\mathrm{r} / \mathrm{d} \approx 0,53 \mathrm{um}$ Zwängungen, aber auch Lebensdauer mindernde Ovalisierungen zu vermeiden,

- Vorzug einer Konstruktion mit Parallelschlaglitzen,

- $\quad$ Sicherstellen, dass Rollen und Führungen leichtgängig und keine Hindernisse vorhanden sind.

Prinzipiell ist auf ein sachgerechtes Handling der Seile vor und während der Montage zu achten. Eine wesentliche Steigerung der Seillebensdauer wird durch Vergrößerung des Scheiben - und Trommeldurchmessers erreicht. Es sollen ein Trommel- und Rollendurchmesser gefunden werden, bei dem das Seil Warrington 6x19+ Stahleinlage mit einem Seilnenndurchmesser $d=3 \mathrm{~mm}$ bei reduziertem Schrägzug auf $\varphi \approx 1,5^{\circ}$ (Vergrößerung Abstand Trommel-Rolle 1 von $1 \mathrm{~m}$ auf ca. $2 \mathrm{~m}$ ) bei guter Schmierung und ohne Schmierung die Ablegereife nach $\mathrm{Z}_{\mathrm{A} 10}=66.000$ Türspielen erreicht (11 Spiele / Stunde x 3 Schichten x 250 Tage). Der Einfluss des Edelstahles wird, wie bereits erwähnt, berücksichtigt.

Um die Ablegespielzahl bei geschmierten Seilen zu erreichen, sollten die Durchmesser von Trommel und Scheiben mindestens $D=200 \mathrm{~mm}$ bei $S=350 \mathrm{~N}$ und mindestens $\mathrm{D}=150 \mathrm{~mm}$ bei $\mathrm{S}=150 \mathrm{~N}$ haben. Bei fehlender Seilschmierung fällt die Seillebensdauer dramatisch ab. Bei trockenen Seilen müsste für den Trommel- und Scheibendurchmesser mindestens $D=300 \mathrm{~mm}$ bei $S=350 \mathrm{~N}$ und mindestens $\mathrm{D}=200 \mathrm{~mm}$ bei $\mathrm{S}=150 \mathrm{~N}$ gewählt werden. Es sei aber angemerkt, dass bei trockenen Seilen die Seillebensdauern stärker streuen als bei gut geschmierten Seilen.

\section{Beispiel 2 - Schleusentorseile - Bauelemente im Stahlwas- serbau der Binnenschifffahrt}

In der modernen Industriegesellschaft ist die Binnenschifffahrt die wirtschaftlichste und umweltfreundlichste Verkehrsart für den Transport von Waren und Personen. Herzstück in der Binnenschifffahrt sind Flüsse und Kanäle zur Überwindung von Höhenunterschieden Schiffshebewerke und Schleusen. Die Tröge der Schiffshebewerke und die Tore der Schleusen werden meist durch Seiltriebe betätigt. Diese Seile müssen sicher und zuverlässig mit allergrößter Verfügbarkeit arbeiten. Schiffsschleusen können eine Hubhöhe bis etwa 25 m haben. In Abbildung 1 wird schematisch der Seiltrieb am Untertor der Schleuse Erlangen des Main-Donau-Kanals dargestellt. Die beiden Gegengewichte sind über Seile, die über die Seilscheiben geführt werden, mit dem Hubtor verbunden. 


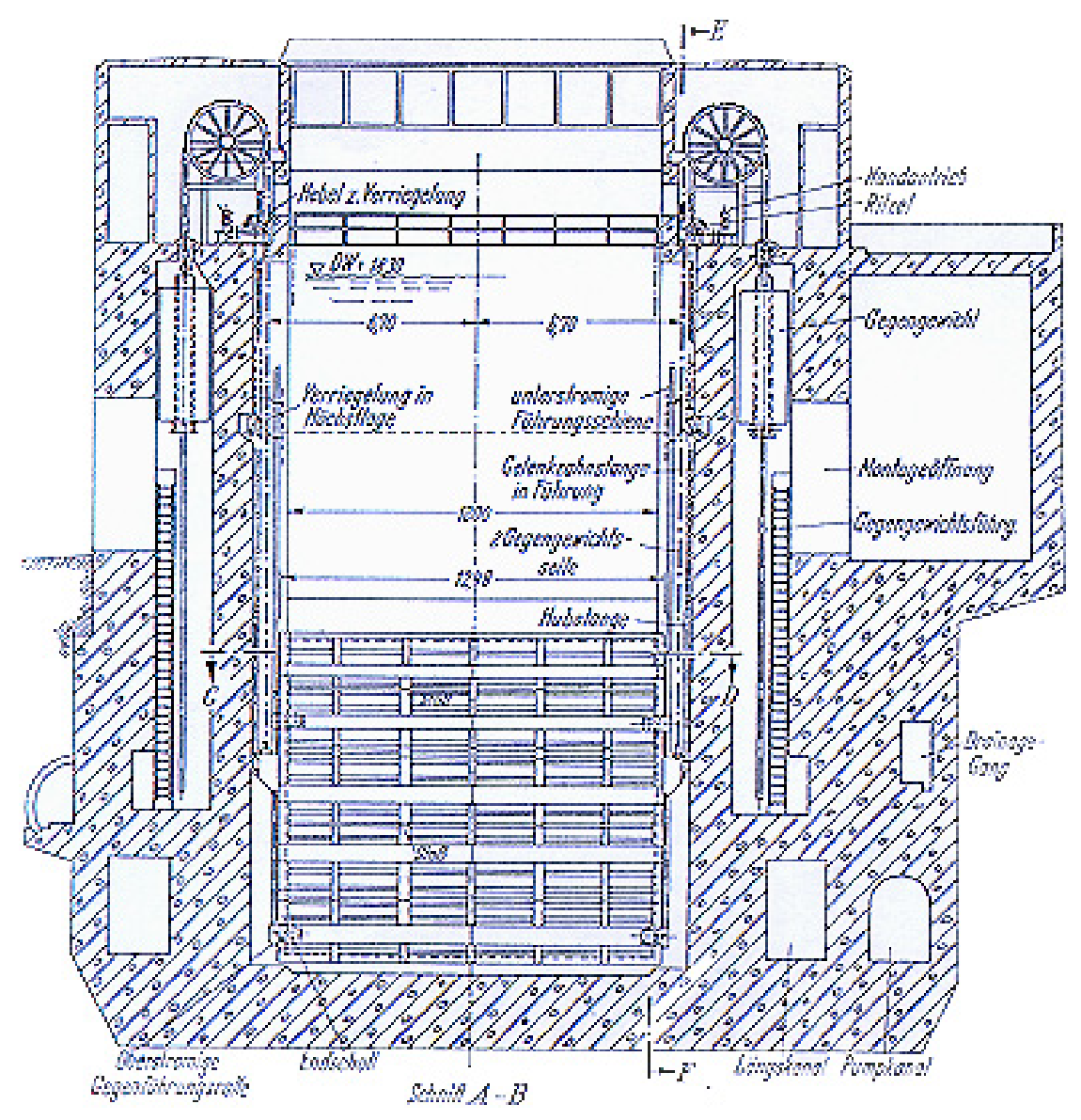

Abbildung 1: Seiltrieb am Untertor der Schleuse Erlangen (Main-Donau-Kanal)

Das Wasser- und Schifffahrtsamt (WSA) Nürnberg ist für den Main-Donau-Kanal auf einer 171 km langen Strecke zwischen Bamberg und Kelheim mit 16 Schleusen für deren Betrieb und Instandhaltung zuständig. Eine zentrale Fragestellung der Verantwortlichen des Wasser- und Schifffahrtsamts (WSA) Nürnberg ist die Lebensdauer und der rechtzeitige Wechsel der Seile vor dem Eintritt eines gefährlichen Zustands. Dieser sicherheitstechnische Aspekt gewinnt zusätzlich an Bedeutung vor dem Hintergrund, dass praktisch keine Redundanz der Seile gegeben ist und beim Bruch eines Seiles am Ober- oder Untertor einer Schleuse schwere Schäden an Sachen und Personen zu erwarten sind. Zum sicherheitstechnischen Aspekt kommt der wirtschaftliche Anspruch, Kosten durch Seilwechsel und Nacharbeit zu minimieren. Basierend auf den langjährigen Erfahrungen des Wasser- und Schifffahrtsamts (WSA) Nürnberg mit den Seiltrieben der Schleusen und in Verbindung mit Ergebnissen aus gezielten Seildauerbiegeversuchen am Institut für Fördertechnik und Logistik (IFT) der Universität Stuttgart, sind Empfehlungen für den Einsatz von Rundlitzenseilen aus Edelstahldrähten und aus verzinkten Drähten aus unlegiertem Kohlenstoffstahl auf stahl- und kunststoffgefütterten Seilscheiben abgeleitet worden. Über die Seildauerbiegeversuche, die Vor- und Nachteile der Seil- und der Scheibenvarianten und der verschiedenen Kombinationen sowie die Grenzen des Einsatzes wird im Folgenden berichtet.

\subsection{Die Frage nach den Seil- und Scheibenwerkstoffen}

Neben den eingangs genannten Sicherheitsaspekten führen wirtschaftliche Gründe durch starken, zum Teil unerwarteten Verschleiß an den Seilen und Scheiben sowie hohe Kosten durch Nacharbeiten an den Stahlscheiben beim Seiltausch zum kritischen Hinterfragen der in den Seiltrieben eingesetzten, verzinkten Stahldrahtseilen und Stahlscheiben. Abbildung 2 zeigt ein stark korrodiertes Seil aus einem Schleusentorantrieb der Schleuse Eibach, das einen umgehenden Austausch notwendig machte. Dieses Seilstück ist beim Stillstand auf der Scheibe gelegen. In Verbindung mit dem aufgenommenen Wasser kam es zu starker Korrosion. In Abbildund 3 ist eine Stahlscheibe mit deutlichen plastischen Verformungen im Rillengrund zu sehen. Diese Seilabdrücke zeigen sich trotz der flammgehärteten Rille mit einer Härteschicht von etwa 5mm Tiefe. Die Nacharbeitung ist zum einen sehr personalintensiv und die Kosten liegen fast in der Größenordnung eines Scheibenneukaufs. 
Zudem sind keine angepassten Werkzeuge für die Nacharbeit verfügbar und Personalausfälle durch Unfälle nicht auszuschließen: Auf eine Nacharbeit der Seilrille und Beseitigung der Verformungsspuren kann aber nicht verzichtet werden, da das Folgeseil unmittelbar nach dem Auflegen Lebensdauer mindernd geschädigt werden könnte.

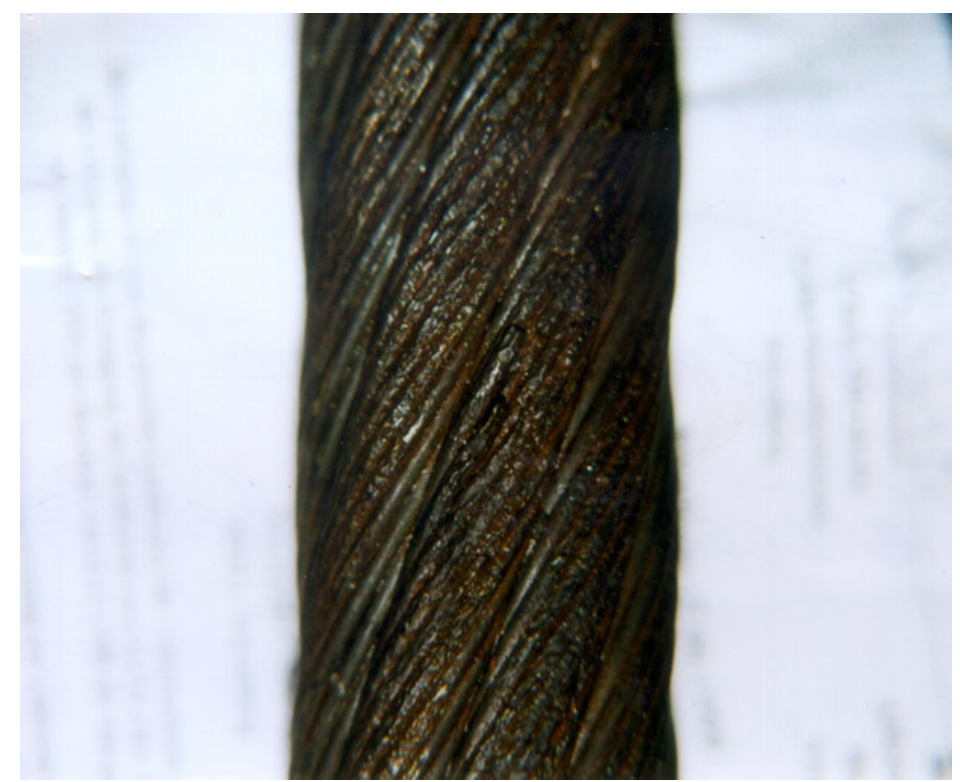

Abbildung 2: korrodiertes, verzinktes Rundlitzenseil - Untertorantrieb der Schleuse Eibach

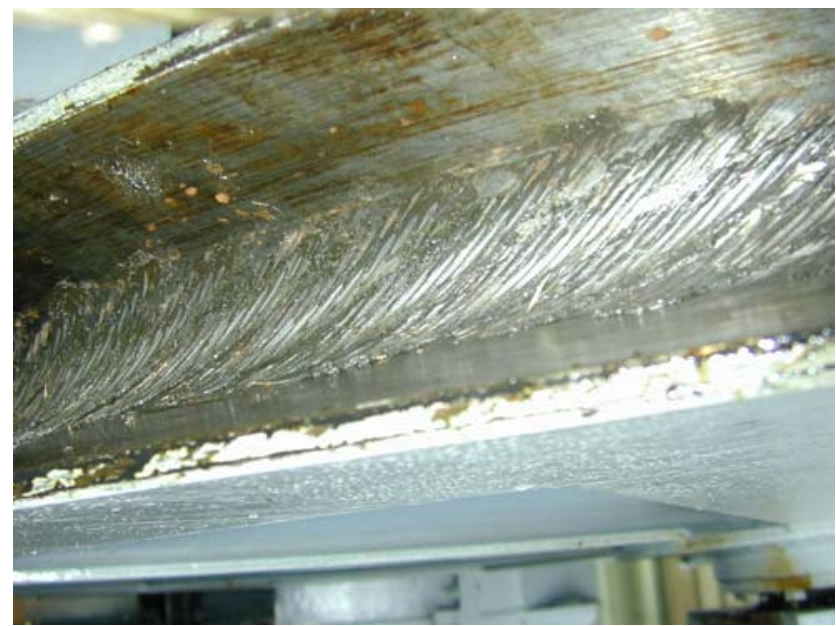

Abbildung 3: Stahlscheibe - Obertorantrieb Schleuse Hippoltstein

Die genannten Erfahrungen mit verzinkten Stahlseilen auf der gehärteten Rille haben dazu geführt, dass

- $\quad$ Drahtseile aus Edelstahldrähten und

- $\quad$ weiche, kunststoffgefütterte Rillenwerkstoffe (hier Becorit) in Verbindung mit verzinkten Stahlseilen und Edelstahlseilen

hinsichtlich der Gebrauchstauglichkeit, d. h. der Eigenschaften beim Lauf über Scheiben und der Sicherheit, untersucht wurden. 


\subsection{Seildauerbiegeversuche}

Beim Öffnen und Schließen der Schleusentore laufen die Seile über die Umlenkscheiben und sind einer kombinierten Beanspruchung von Zug, Biegung, sekundären Spannungen und Pressungen unter harten Umgebungsbedingungen ausgesetzt.

Dieser Lauf der Seile über Scheiben kann im Labor auf Dauerbiegemaschinen zeitraffend bis zum Seilbruch simuliert werden. Für die Dauerbiegeversuche wird die Dauerbiegemaschine 5 des IFT eingesetzt, (s. Abb.4). Bei der Dauerbiegemaschine ist das Versuchsseil um die obere Umlenkscheibe und um die eigentliche, unten befindliche Seilprüfscheibe gelegt. Die Prüfscheibe führt eine oszillierende Bewegung mit vorgegebenem Hub durch. Das Versuchsseil gelangt dabei vom geraden in den gekrümmten und wieder zurück in den geraden $\mathrm{Zu}-$ stand und wird dabei durch Biegewechsel beansprucht. Während des Dauerbiegeversuchs ist das Seil über den an der oberen Umlenkscheibe angreifenden Hydraulikzylinder mit einer konstanten Zugkraft vorgespannt. Der Dauerbiegeversuch ist wegen der konstanten Seilzugkraft bestens zur Untersuchung der Biegewechseleigenschaften geeignet, da auch im Seiltrieb des Schleusentores das Seil einer ständigen, konstanten Last ausgesetzt ist.

\subsection{Seile}

Für den Einsatz in den Schleusentorantrieben und in den Dauerbiegeversuchen sind Rundlitzenseile der Konstruktion Filler 8x19 mit Fasereinlage mit einem Seilnenndurchmesser d=40mm vorgesehen. Die Drahtwerkstoffe sind unlegierter, hochgekohlter Kohlenstoffstahl oder Edelstahl mit der Werkstoffnummer 1.4401. Im Folgenden werden für die Seile die Abkürzungen „Edelstahlseil“ und „Normalstahlseil“ verwendet. Die Seildaten der beiden Seile nach Angaben des Seilherstellers sind in Tabelle 4 zusammengefasst.

Tabelle 4: Seildaten

\begin{tabular}{|c|c|c|}
\hline & Edelstahlseil & Normalstahlseil \\
\hline Seil $\varnothing \mathrm{d}_{\mathrm{Nenn}} \quad[\mathrm{mm}]$ & 40 & 41 \\
\hline Seilkonstruktion & \multicolumn{2}{|c|}{ Filler 8x19 FE } \\
\hline Schlagart und Richtung & \multicolumn{2}{|c|}{ Gleichschlag zZ } \\
\hline Drahtmaterial & Edelstahl 1.4401 & Unlegierter Kohlenstoffstahl \\
\hline Drahtnennfestigkeit $\mathrm{R}_{\mathrm{o}} \quad\left[\mathrm{N} / \mathrm{mm}^{2}\right]$ & 1570 & 1970 \\
\hline Metall. Seilquerschnitt ${ }^{(*)} \mathrm{A} \quad\left[\mathrm{mm}^{2}\right]$ & 559 & $587^{(*)}$ \\
\hline Bruchkraft $\quad$ Ermittelte $\mathrm{F}_{\mathrm{e}}$ & 938 & - \\
\hline Wirkliche $\mathrm{F}_{\min }$ & 860 & $1114,1120,1121$ \\
\hline Schmierung & \multicolumn{2}{|c|}{ Anlieferungszustand } \\
\hline
\end{tabular}

*mit dem Nenndurchmesser und dem Füllfaktor aus DIN 3051 


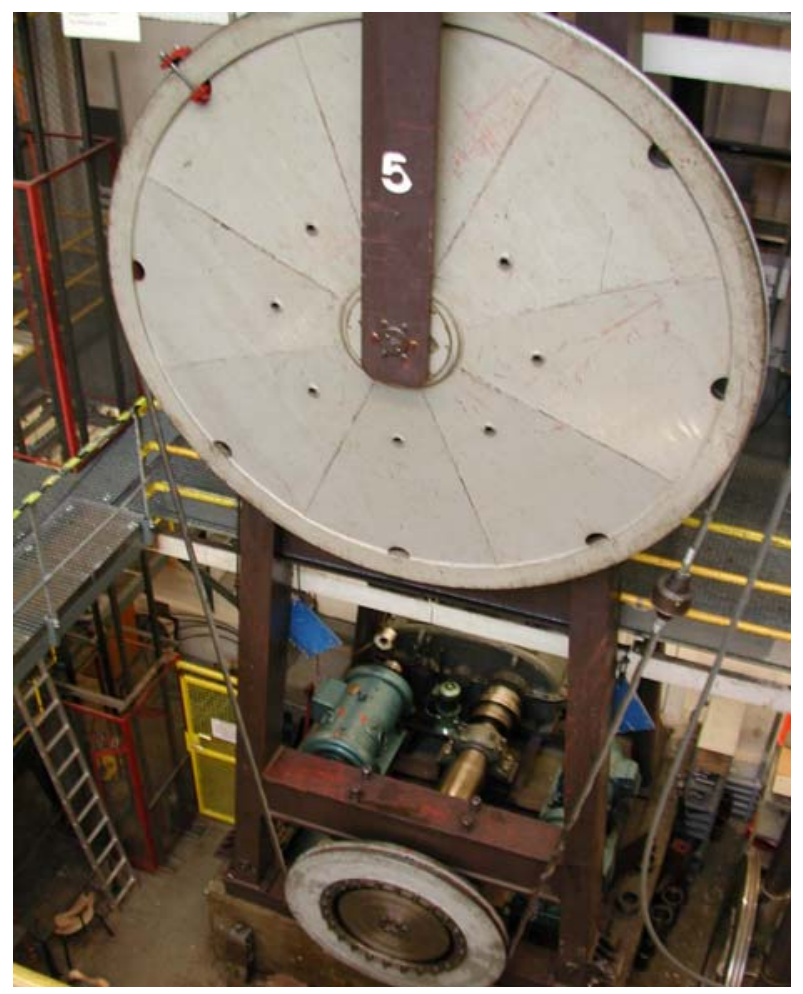

Abbildung 4: Dauerbiegeversuch mit Schleusentorseilen

\subsection{Seilscheibe}

Für die Dauerbiegeversuche ist eine Originalscheibe aus einem Schleusentorantrieb für den Einbau in die Seildauerbiegemaschine 5 umgearbeitet worden. Die Seilrille ist als Rundrille ausgeführt, Abbildung 5. Das Rillenmaterial ist mit einem Kunststoff der Firma Becorit ausgestattet. Die Daten der Versuchsscheibe und der Rille sind in Tabelle 5 zusammengefasst.

Tabelle 5: Daten der Versuchsscheibe

\begin{tabular}{|l|c|}
\hline $\begin{array}{l}\text { Seilprüfscheibendurchmesser Seilmitte- } \\
\text { Seilmitte D }\end{array}$ & 1538 \\
\hline Rillenm] & 21,2 \\
\hline Durchmesserverhältnis D/d $\mathrm{r}[\mathrm{mm}]$ & $\approx 40$ \\
\hline Scheibenwerkstoff & Becurit \\
\hline
\end{tabular}

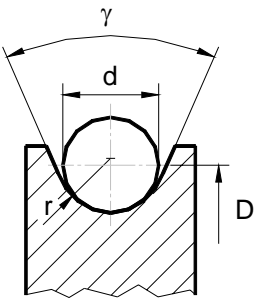

Rundrille

Abbildung 5: Rille der Prüfscheibe 


\subsection{Versuchdaten der Dauerbiegeversuche und Durchführung}

Die Versuchsdaten sind in Tabelle 3 zusammengefasst. Die gewählte Seilzugkraft von S=200kN entspricht der im praktischen Betrieb des Schleusentores auftretenden Seilzugkraft, die durch die Gewichtskraft eines Gegengewichtes bzw. des Schleusentores auftritt. Die größere untersuchte Seilzugkraft liefert in kürzerer Versuchszeit einen zweiten Versuchswert und erleichtert so den Vergleich mit vorhandenen Erkenntnissen, z. B. aus Feyrer [Feyrer00]

Tabelle 6: Versuchsdaten

\begin{tabular}{|l|c|c|}
\hline Seilzugkraft S [kN] & 200 & 300 \\
\hline Seilhub h $[\mathrm{m}]$ & 1200 \\
\hline Biegelänge l $[\mathrm{mm}]$ & $\approx 2 \times 1200$ \\
\hline Biegewechsel pro Stunde [N/h] & 400 \\
\hline Schmierung & Anlieferungszustand \\
\hline
\end{tabular}

Während des Dauerbiegeversuchs werden auf den zwei Biegezonen von jeweils $30 \mathrm{x}$ d die Drahtbrüche gezählt und die Seildurchmesser gemessen. Außerdem wird auf beiden Biegezonen die maximale Drahtbruchzahl auf einer Bezugslänge von 6 x d ermittelt.

\subsection{Bruchbiegewechselzahlen}

Die im Dauerbiegeversuch ermittelten Bruchbiegewechselzahlen für die beiden Versuchsseile sind in Tabelle 7 zusammengefasst. Mit dem Edelstahlseil sind 2 Dauerbiegeversuche bei einer Seilzugkraft S=200kN durchgeführt worden. Die Bruchbiegewechselzahlen sind in Abbildung 6 dargestellt.

Tabelle 7: Bruchbiegewechselzahlen

\begin{tabular}{|c|c|c|c|c|}
\hline & \multicolumn{2}{|c|}{ Edelstahlseil } & \multicolumn{2}{c|}{ Normalstahlseil } \\
\hline Seilzugkraft S [KN] & 200 & 300 & 200 & 300 \\
\hline Bruchbiege-wechselzahl N & 270.510 & 127.910 & 1.099 .430 & 340.590 \\
& 178.690 & & & \\
\hline
\end{tabular}

Zusätzlich sind in Abbildung 6 die errechneten Biegewechselzahlen nach Feyrer 2000 für die untersuchte Seilkonstruktion aus Normalstahl eingezeichnet. Im vorliegenden Fall sind kunststoffgefütterte, weiche Rillen eingesetzt worden. Die Biegewechselzahlen beim Lauf der Seile in weichen Rillen sind größer als beim Lauf in der Stahlrille. Der Zuwachs an Lebensdauer ist von Feyrer 2000 beschrieben worden. Die nach Feyrer 2000 nach oben korrigierten, errechneten Biegewechselzahlen sind ebenfalls in Abbildung 6 eingezeichnet. 


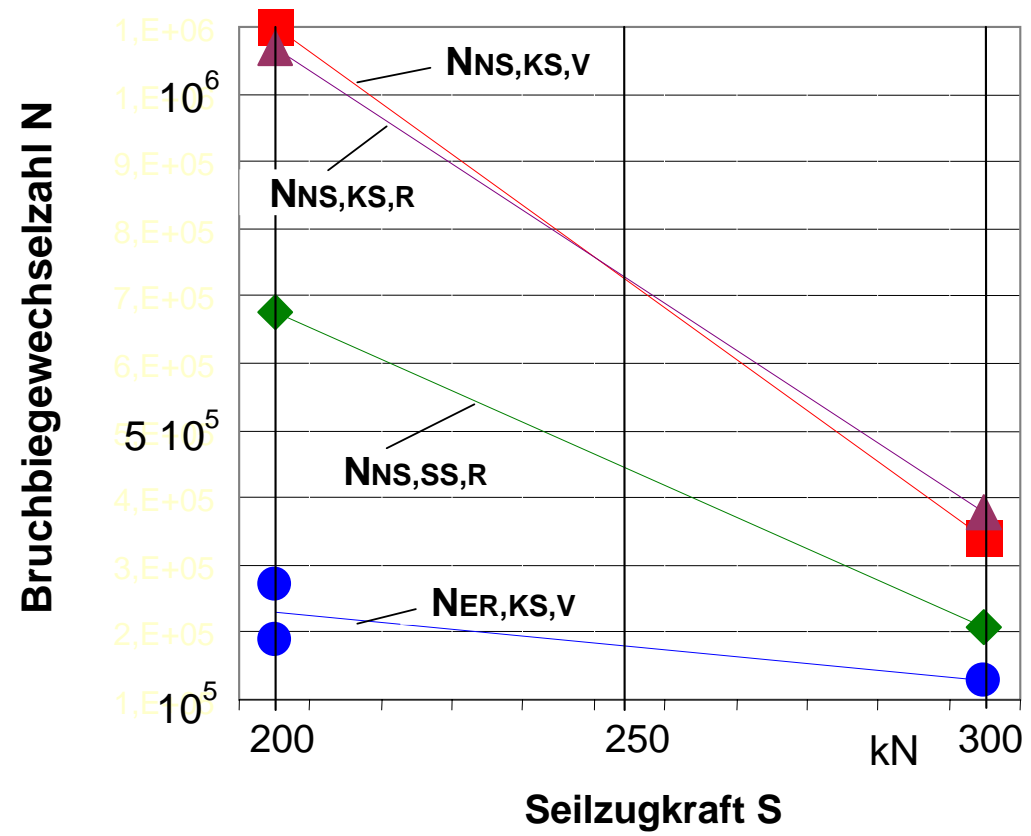

Abbildung 6: Bruchbiegewechselzahlen aus Dauerbiegeversuchen und Berechnung

$\mathrm{N}_{\mathrm{ER}, \mathrm{KS}, \mathrm{V}}$ : Bruchbiegewechselzahl Edelstahlseil auf Kunststoffscheibe (Versuch)

$\mathrm{N}_{\mathrm{NS}, \mathrm{KS}, \mathrm{V}}$ : Bruchbiegewechselzahl Normalstahlseil auf Kunststoffscheibe (Versuch)

$\mathrm{N}_{\mathrm{NS}, \mathrm{SS}, \mathrm{R}}$ : Bruchbiegewechselzahl Normalstahlseil auf Stahlscheibe (Rechnung)

$\mathrm{N}_{\mathrm{NS}, \mathrm{KS}, \mathrm{R}}$ : Bruchbiegewechselzahl Normalstahlseil auf Kunststoffscheibe (Rechnung)

\subsection{Ablegekriterien}

Als Ablegekriterien bei über Scheiben laufenden Seilen können Drahtbrüche, Veränderungen des Seildurchmessers, grobe Seilschäden, in besonderen Fällen die Aufliegezeit etc. herangezogen werden. Die Kriterien zur Ermittlung der Ablegereife werden im Verlauf der Dauerbiegeversuche bei den regelmäßigen Inspektionen ermittelt. Maßgebend für die Ablegereife ist die Anzahl von Drahtbrüchen auf Bezugslängen auf dem Seil. Die Anzahl der Drahtbrüche auf Bezugslängen ist in DIN 15020 in Abhängigkeit der Triebwerksgruppe, der Seilkonstruktion und deren Drahtfestigkeit geregelt, und zwar streng genommen für den Lauf der Seile über Stahlscheiben. Unter der Annahme einer mittleren täglichen Laufzeit zwischen einer und zwei Stunden sind die Triebwerksgruppen $2 \mathrm{~m}$ bis $5 \mathrm{~m}$ maßgebend mit den Ablegedrahtbruchzahlen für Gleichschlagseile von 13 Drahtbrüchen auf einer Länge von 30 x d und 6 Drahtbrüchen auf einer Länge von 6 x d.

\section{Edelstahlseile}

Bei den Dauerbiegeversuchen mit den Edelstahlseilen treten nur sehr wenige oder keine äußerlich sichtbaren Drahtbrüche in den Biegezonen auf. Der Seildurchmesser hat sich im Verlauf der Dauerbiegeversuche praktisch nicht verändert. Die Entwicklung der Drahtbrüche und der Seildurchmesser können damit nicht als Ablegekriterium herangezogen werden. Als Ablegekriterium soll die Biegewechselzahl herangezogen werden. Bisher liegen erst Ergebnisse aus drei Dauerbiegeversuchen mit den im Schleusenbetrieb eingesetzten Edelstahlseilen vor. Wegen der geringen Anzahl an Versuchen, den großen Unterschieden bei den erreichten Biegewechselzahlen und den nur geringen Erfahrungen von über Scheiben laufenden Edelstahlseilen kann die Biegewechselzahl mit einer sehr vorsichtigen Abgrenzung als Ablegekriterium herangezogen werden. Für diese Abgrenzung wird angenommen, dass die Biegewechselzahl $\mathrm{N}=270.510$ bei $\mathrm{S}=200 \mathrm{kN}$ einer bestimmten Ausfallsummenhäufigkeit zugeordnet wird. Die Standardabweichungen der Ergebnisse aus den Dauerbiegeversuchen an Normalstahlseilen aus einer Charge werden angesetzt für den schlechtesten Fall, d. h. auf der sicheren Seite. Mit diesen Annahmen wird bei einer Ausfallsummenhäufigkeit $\phi(u)=99 \%$ die Biegewechselzahl nur von 1\% übertroffen. Bei der Abschätzung mit $\phi(\mathrm{u})=99 \%$ wird mit dem Standardisierungsparameter $\mathrm{u}=2,326$ der fiktive Mittelwert und mit $2 \mathrm{u}$ die Biegewechselzahl für die Ausfallsummenhäufigkeit $\phi(\mathrm{u})=1 \%$ berechnet, d. h. der Biegewechselzahl, die in $1 \%$ der Fälle nicht erreicht wird. Beispielhaft ist die durch $\phi(\mathrm{u})=1 \%$ abgegrenzte Biegewechselzahl, berechenbar mit $\lg \mathrm{N}_{1 \%}=\lg \mathrm{N}_{99 \%}-2 \cdot \mathrm{u} \cdot$ lgs. Die bei $1 \%$ abgegrenzte Biegewechselzahl ist $\mathrm{N}_{1 \%}=120.000$.

Die Biegewechselzahl $\mathrm{N}_{1 \%}=120.000$ ergibt bei etwa 6500 Schleusungen pro Jahr und zwei Biegewechseln pro Schleusung (d.h. etwa 13.000 Biegewechsel pro Jahr) einer Nutzungsdauer von etwa 10 Jahren. 


\section{Normalstahlseile}

Durch regelmäßige Seilinspektionen während der Dauerbiegeversuche sind in den Biegezonen äußerlich sichtbare Drahtbrüche festgestellt worden. Die Ablegereife wurde jedoch nicht durch äußerlich sichtbare Drahtbrüche nach DIN 15020 erkannt. DIN 15020 ist auf den Einsatz von Stahlscheiben abgestimmt und sieht den Fall einer kunststoffgefütterten Scheibe nicht vor. Für den Seiltrieb des Schleusentores wurde die Ablegebiegewechselzahl daher auf 5 Drahtbrüche auf einer Länge von $30 \mathrm{x}$ d und 3 Drahtbrüche auf einer Länge von $6 \mathrm{x}$ d gegenüber DIN 15020 deutlich reduziert. Die Ablegebiegewechselzahl beträgt damit unter Praxisbedingungen etwa 1/3 der Bruchbiegewechselzahl.

\subsection{Einsatzmöglichkeiten der Varianten Seilscheibe}

Bei der gehärteten Stahlscheibe nach dem Einsatz treten trotz der flammgehärteten Rillenoberfläche plastische Verformungen im Rillengrund auf. Ohne Nacharbeit würde das neue Seil unmittelbar nach dem Auflegen geschädigt und müsste vorzeitig getauscht werden. Eine gebrauchte Stahlscheibe muss folglich nachgearbeitet werden, d.h.es muss eine „Abdruckfreiheit“ sichergestellt werden, um die zu erwartende Lebensdauer auch tatsächlich zu erreichen. Aus den Erfahrungen des WSA Nürnberg und den Ergebnissen der Dauerbiegeversuche ergeben sich folgende sinnvolle Kombinationen von Seilwerkstoff und Scheibenmaterial, aufsteigend nach der zu erwartenden Lebensdauer zusammengestellt:

1. Normalstahlseil auf Stahlscheibe,

2. Edelstahlseil auf Stahlscheibe,

3. Edelstahlseil auf kunststoffgefütterter Scheibe und

4. Normalstahlseil auf kunststoffgefütterter Scheibe.

Aufgrund der Ergebnisse der Dauerbiegeversuche und der Entwicklung des Hauptablegekriteriums Drahtbrüche ist die Einsatzdauer begrenzt. Wegen der relativ genau prognostizieren Anzahl der Schleusungsvorgänge innerhalb eines Jahres ist vom Wasser- und Schifffahrtsamt die Einsatzzeit bis zum Ablegen der Seile bei der Variante Edelstahlseil auf kunststoffgefütterter Scheibe auf 10 Jahre und bei der Kombination Normalstahlseil auf kunststoffgefütterter Scheibe auf 20 Jahre begrenzt worden. Die Einsatzzeiten der Varianten 1 bis 3 liegen deutlich unterhalb der Variante 4.

Bei den Seilen in Schleusentorantrieben werden metallische Vergüsse bzw. Kunststoffvergüsse eingesetzt. Bei den Normalstahlseilen ist die Wahl der Vergussmasse freigestellt. Bei den Edelstahlseilen ist aber nur der Kunststoffverguss zugelassen. Nur mit dieser Vergussmasse ist eine zuverlässige Verbindung des Systems Seildraht, Vergussmasse und Vergusshülse sichergestellt. Die folgende Zusammenstellung der Vor- und Nachteile des Einsatzes von Edelstahlseilen gegenüber Normalstahlseilen gibt eine Entscheidungshilfe für den geeigneten Einsatz in Abhängigkeit der Randbedingungen in den unterschiedlichen Schleusentorantrieben.

\section{Vorteil von Edelstahlseilen gegenüber Seilen aus Drähten aus Kohlenstoffstahl (Normalstahlseile)}

- Korrosionswiderstand allgemein höher als bei Normalstahlseilen

- Korrosion infolge Kontakt mit Stahlrille vernachlässigbar

- Mit dem Kunststoffverguss kleine Streuung bei der Seilbruchkraft und Seilbruch stets auf freier Strecke

- Keine Lebensdauerbeeinflussung durch Korrosionseffekte zu erwarten

\section{Nachteil von Edelstahlseil gegenüber Seilen aus Drähten aus Kohlenstoffstahl (Normalstahlseile)}

- Empfindlicher gegen Beschädigung beim Auflegen der Seile

- Kleinere Lebensdauer beim Lauf über kunststoffgefütterte Scheiben

- Höhere Kosten beim Seil und des Kunststoffvergusses

- $\quad$ Geringeres Einsatztemperaturfeld

Vom Wasser- und Schifffahrtsamt Nürnberg ist berichtet worden, dass infolge der Wasseraufnahme bei jedem Schleusengang Korrosion an den verzinkten Seilen in Kombination mit unaufgefütterten Stahlseilscheiben auftritt. Bei Korrosion an den verzinkten Seilen, bedingt durch Wasseraufnahme, ist - wie auch allgemein im Gebrauch und bei Verschleiß - ein Bruchkraftverlust zu erwarten.

Durch den Gebrauch, die Korrosion und Verschleiß an den verzinkten Seilen wird die Sicherheit der Seile gegen Bruch im geraden Strang vermindert. Bei den Edelstahlseilen hingegen ist kein Bruchkraftverlust durch Korrosion infolge Wasseraufnahme zu erwarten. 
Mit Drahtseilen der Konstruktion Filler 8x19 mit Fasereinlage und Drähten aus Edelstahl (Edelstahlstahlseil) oder aus Drähten aus unlegiertem Kohlenstoffstahl (Normalstahlseil) sind Dauerbiegeversuche am IFT auf einer kunststoffgefütterten Scheibe aus einem Schleusentorantrieb des Main-Donau-Kanals unter Berücksichtigung der dort herrschenden Seilkräfte durchgeführt worden. Die Lebensdauer der Normalstahlseile ist größer als die der Edelstahlseile, wobei eine Beeinflussung der Lebensdauer bei dem Edelstahlseil infolge Korrosion auszuschließen ist. Auf der Basis der Ergebnisse der Dauerbiegeversuche ist für das Edelstahlseil auf der Basis der ermittelten Bruchbiegewechselzahlen mit statistischer Abgrenzung eine Ablegebiegewechselzahl festgelegt worden. Bei den Normalstahlseilen ist eine Ablegedrahtbruchzahl auf Bezugslängen empfohlen worden. Der Einsatz von Normalstahlseilen auf „weicher“ kunststoffgefütterter Seilscheibe ist die wirtschaftlichste Kombination mit der längsten Lebensdauer und Standzeit. Ist jedoch ein nachträgliches „Ausfüttern“ der vorhandenen „harten“ Seilscheiben nicht möglich, können Edelstahlseile im Wasserwechseleinsatz die längsten Standzeiten erreichen.

\section{Aktuelle Forschung auf dem Gebiet der laufenden Edel- stahlseile}

Das AiF-Forschungsprojekt Nr. 14501 N „Laufende Edelstahlseile“ wird aus Haushaltsmitteln des Bundesministeriums für Wirtschaft und Arbeit BMWA über die Arbeitsgemeinschaft industrieller Industrievereinigungen „Otto von Guericke“ e.V. (AiF) gefördert. Für das Forschungsvorhaben wurden 10 Versuchsseile ausgewählt. Mit den 10 ausgewählten Versuchsseilen werden vier unterschiedliche Seilkonstruktionsklassen (6x36 Warrington-SealeS, 6x19 Standard, 6x19 Seale, 18x7) und sehr unterschiedliche Seilnenndurchmesser von 1,5 mm bis $14 \mathrm{~mm}$ und verschiedene Seilhersteller berücksichtigt, um grundlegende und gesicherte Erkenntnisse über die Lebensdauer von Seilen aus Edelstahldrähten zu schaffen. Als Drahtwerkstoff steht der Edelstahl mit der Bezeichnung 1.4401 im Vordergrund, wobei auch die Werkstoffe 1.4301 und 1.4436 im Rahmen der Untersuchung geprüft werden sollen.

\subsection{Versuchsparameter}

Bei den umfangreichen Versuchsreihen werden Dauerbiegeversche bei Raumtemperatur und unter NaClEinfluss durchgeführt. Im Fokus steht die Schaffung einer Datenbasis für die spätere Ableitung einer Lebensdauergleichung für Edelstahlseile. Weiterhin werden Versuche bei hohen Seilzugkräften durchgeführt, um die so genannte Sprungspannung zu ermitteln.

In speziellen Versuchsreihen wird der Einfluss der Schmierung auf die Biegewechselleistung der Edelstahlseile untersucht. Flankiert werden die Dauerversuche durch herkömmliche Drahtprüfungen wie Zugversuch und Hinund Herbiegeversuch sowie metallographische und fraktographische Untersuchungen.

\subsection{Bisher erzielte Versuchsergebnisse}

In den Dauerbiegeversuchen werden die verschiedenen Einflussparameter so variiert, dass eine Basis für eine angepasste Lebensdauergleichung für die Edelstahlseile abgeleitet werden kann, vergleichbar der bei Kohlenstoffstahlseilen gängigen und bewährten Vorgehensweise.

Bisher sind Versuchsergebnisse von drei Seilkonstruktionsklassen verfügbar. Die Versuche wurden dabei regelmäßig nach der Renard-Reihe angehalten und die Seile inspiziert. Damit wird die Seilschädigung über der Biegwechselzahl, d. h. die Entwicklung der äußerlich sichtbaren Drahtbruchzahlen und der Seildurchmesserverlust, ermittelt. Abbildung 7 zeigt, dass die Versuchsergebnisse der bisher untersuchten 6x36 Warrington-Seale-Seile unterschiedlicher Hersteller bei nur gering von einander abweichenden Seildurchmessern von 12 mm und 14 mm stark korrelieren und gemeinsam in einer Seilkonstruktionsklasse ausgewertet werden können. 

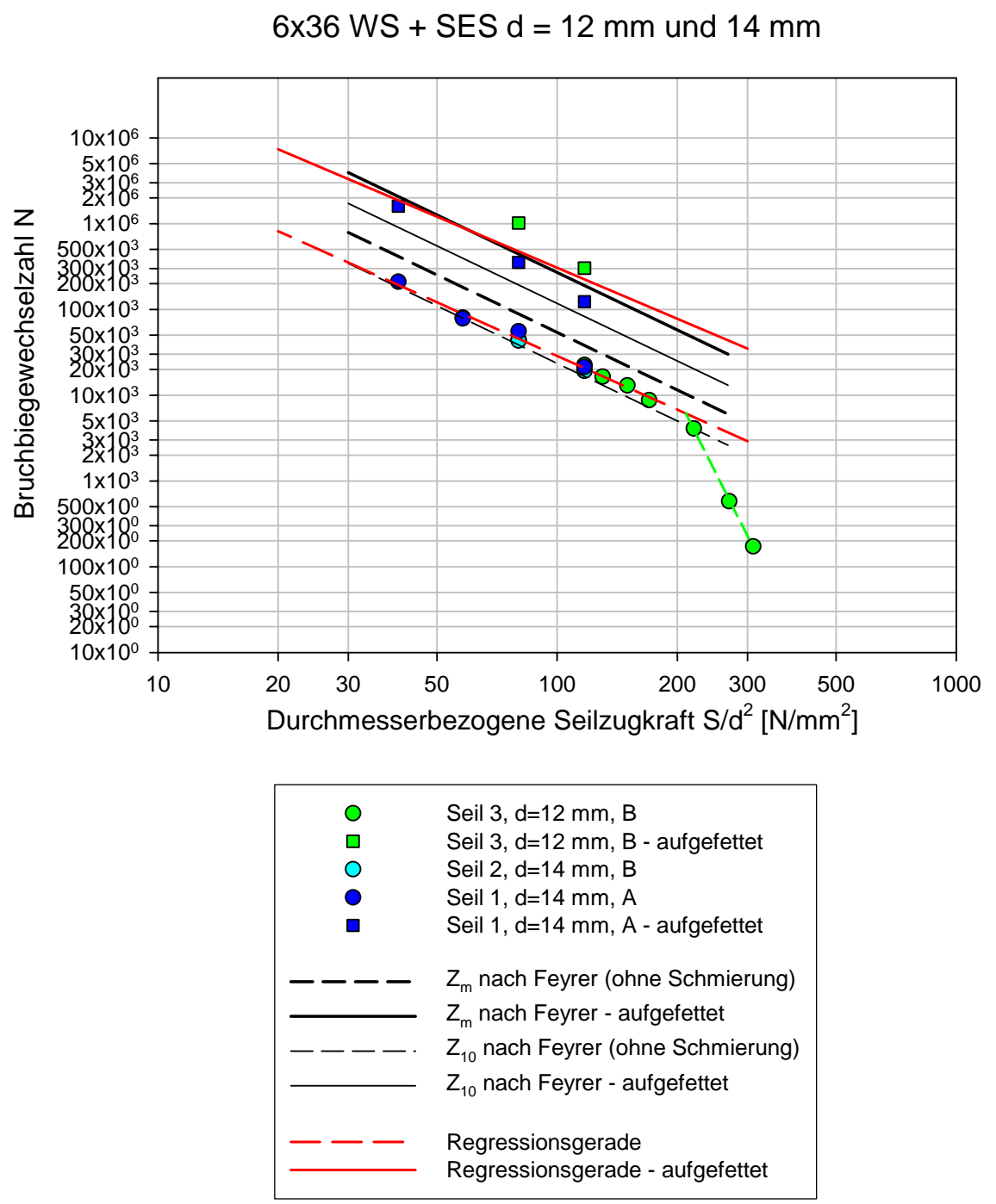

Abbildung 7: Ermittelte Bruchbiegewechselzahlen von Edelstahlseilen 6x36 WS + IWRC (1.4401)

\section{Zusammenfasung}

Die Biegewechseleigenschaften von laufenden Edelstahlseilen ist bisher nur wenig untersucht. Die beiden angeführten Beispiele zeigen, dass großer Forschungsbedarf auf dem Gebiet der laufenden Edelstahlseile besteht. Alarmiert durch die Ausfälle an fördertechnischen Anlagen und auch Unfällen ist eine groß angelegtes Forschungsprojekt angeregt worden, um sicherheitstechnische Lücken zu schließen aber auch um wirtschaftlichen Schaden im Vorfeld abwenden zu können.

\section{Literatur}

[Feyrer00] Feyrer, K.: Drahtseile. Berlin [u.a.]: Springer 2000. 